\title{
Testing various polarized parton distributions at RHIC
}

\author{
Jacques Soffer ${ }^{1}$ and Jean Marc Virey ${ }^{2, a}$ \\ ${ }^{1}$ Centre de Physique Théorique*, C.N.R.S. - Luminy, Case 907 \\ F-13288 Marseille Cedex 9, France \\ ${ }^{2}$ Centre de Physique Théorique and Université de Provence, Marseille, France
}

\begin{abstract}
A very promising spin physics programme will be soon on the way at the BNL Relativistic Heavy Ion Collider (RHIC). By studying the spin asymmetries for various processes (single photon, single-jet and $W^{ \pm}$production), we will compare the different predictions obtained using some sets of polarized parton distributions, available in the recent literature. We will put some emphasize on the analysis of the anticipated errors, given the event rates expected from this high luminosity new machine and the current acceptance for the detector systems at RHIC.
\end{abstract}

PACS Numbers : Key-Words :

Number of figures : 14

May 1997

CPT-97/P.3495

anonymous ftp or gopher : cpt.univ-mrs.fr

*Unité Propre de Recherche 7061

${ }^{a}$ Moniteur CIES and allocataire MESR

E-mail : Soffer@cpt.univ-mrs.fr ; Virey@cpt.univ-mrs.fr 


\section{Introduction}

Serious progress have been made over the last ten years or so, in our understanding of the spin structure of the nucleon. This is partly due to the discovery in 1987, by the EMC experiment [1], of the violation of the Ellis-Jaffe sum rule [2]. This result was obtained by measuring the proton polarized structure function $g_{1}^{p}\left(x, Q^{2}\right)$, in polarized deep-inelastic scattering with a polarized amonia target $\left(\mathrm{NH}_{3}\right)$. It led to an unexpected situation, that is, only a small fraction of the proton spin is carried by quarks, which is at variance with the naive quark-parton model. The EMC result has motivated several experiments, in three different laboratories CERN, DESY and SLAC [3], to undertake some systematic determinations of $g_{1}^{p, n}\left(x, Q^{2}\right)$, for proton and neutron by means of measurements using a polarized lepton beam and several different polarized targets (hydrogen, deuterium, helium-3). The main goal of these experimental programmes was to confirm the EMC result on $g_{1}^{p}\left(x, Q^{2}\right)$ and to measure the neutron structure function $g_{1}^{n}\left(x, Q^{2}\right)$, in order to test the fundamental Bjorken sum rule [4]. It turns out that these fixed target experiments allow to cover only a rather limited kinematic domain that is, $0.005<x<0.7$, with the corresponding average $Q^{2},<Q^{2}>$ between $2 \mathrm{GeV}^{2}$ and $10 \mathrm{GeV}^{2}$. Needless to recall that the low $x$ region, which is not easily accessible and therefore loosely determined, is very crucial for testing accurately these sum rules. In addition it is not possible to make a flavor separation to isolate the contribution of each quark to the nucleon spin.

On the theoretical side, the surprising EMC result has also stimulated an intense activity, in particular its possible interpretation in terms of the so-called axial anomaly which involves the gluon polarization $\Delta G\left(x, Q^{2}\right)$ [5, 6, 7]. This is an interesting physical quantity in itself, but it can only be determined indirectly in polarized deep-inelastic scattering. More precisely the contribution of the gluon polarization to the Ellis-Jaffe sum rules is ambiguous and, as we will see below, much cleaner information will be extracted at the RHIC facility from collisions with polarized proton beams. Let us also mention that several important results in higher order perturbative QCD corrections have been obtained recently [5, 6, 7], which will allow, in the future, very stringent tests of the QCD spin sector, so far essentially unexplored.

In order to describe the most recent experimental results on $g_{1}^{p, n, d}\left(x, Q^{2}\right)$, several phenomenological models have been proposed and correspondingly, one can find in the recent literature various sets of parametrization for the polarized parton distributions. In spite of the constant progress in the accuracy of the data, the extraction of these distributions, especially for sea quarks and gluons, remains fairly ambiguous and controversial. We will select some of them and recall their main features in Section 2, where we also give some basic kinematics and the definitions of the spin observables we will study below. In Section 3 we will consider direct photon production. In terms of perturbative QCD, it is a simple process whose double helicity asymmetry is particularly sensitive to $\Delta G\left(x, Q^{2}\right)$, as we will illustrate by means of a useful approximate expression. Section 4 is devoted to single-jet production which has a higher complexity than the previous process but whose generous cross section will allow a very accurate determination of the double helicity asymmetry. We will consider in Section 5 the production of $W^{ \pm}$gauge bosons and parity 
violating asymmetries with either one or two polarized beams. Their measurements will provide a good calibration of the quark and antiquark polarizations and also the $u$ and $d$ flavor separation. We will comment on the possible benefit one can get by measuring them simultaneously in proton-proton and proton-neutron collisions. We will give our concluding remarks in Section 6.

\section{Spin observables and various parametrizations}

Fundamental interactions at short distances which are probed in high energy hadronic collisions, involve hard scattering of quarks, antiquarks and gluons. Let us consider the general hadronic reaction

$$
a+b \rightarrow c+X
$$

where $c$, in the cases we will consider below, is either a photon, a $W^{ \pm}$or a single-jet. In the hard scattering kinematic region, the cross section describing (1) reads in the QCD parton model, provided factorization holds, as

$$
d \sigma(a+b \rightarrow c+X)=\sum_{i j} \frac{1}{1+\delta_{i j}} \int d x_{a} d x_{b}\left[f_{i}^{(a)}\left(x_{a}, Q^{2}\right) f_{j}^{(b)}\left(x_{b}, Q^{2}\right) d \hat{\sigma}^{i j}+(i \leftrightarrow j)\right] .
$$

The summation runs over all contributing parton configurations, the $f\left(x, Q^{2}\right)$ 's are the parton distributions, directly extracted from deep-inelastic scattering for quarks and antiquarks and indirectly for gluons. $\mathrm{d} \hat{\sigma}_{i j}$ is the cross section for the interaction of two partons $i$ and $j$ which can be calculated perturbatively, some of which, at the lowest order, are given in ref. [8]. If we consider the reaction (1) with both initial hadrons, $a$ and $b$ longitudinally polarized, one useful observable is the double helicity asymmetry $A_{L L}$ defined as

$$
A_{L L}=\frac{d \sigma_{a(+) b(+)}-d \sigma_{a(+) b(-)}}{d \sigma_{a(+) b(+)}+d \sigma_{a(+) b(-)}},
$$

when we assume parity conservation, i.e. $\mathrm{d} \sigma_{a(\lambda) b\left(\lambda^{\prime}\right)}=\mathrm{d} \sigma_{a(-\lambda) b\left(-\lambda^{\prime}\right)}$. Its explicit expression, assuming factorization, is given by

$$
A_{L L} d \sigma=\sum_{i j} \frac{1}{1+\delta_{i j}} \int d x_{a} d x_{b}\left[\Delta f_{i}^{(a)}\left(x_{a}, Q^{2}\right) \Delta f_{j}^{(b)}\left(x_{b}, Q^{2}\right) \hat{a}_{L L}^{i j} d \hat{\sigma}^{i j}+(i \leftrightarrow j)\right],
$$

where $\mathrm{d} \sigma$ is given by eq.(2) and $\hat{a}_{L L}^{i j}$ denotes the corresponding subprocess double asymmetry for initial partons $i$ and $j$. The $\Delta f$ 's are defined as

$$
\Delta f\left(x, Q^{2}\right)=f_{+}\left(x, Q^{2}\right)+f_{-}\left(x, Q^{2}\right)
$$

where $f_{ \pm}$are the parton distributions in a polarized hadron with helicity either parallel $(+)$ or antiparallel (-) to the parent hadron helicity. Recall that the unpolarized distributions are $f=f_{+}+f_{-}$and $\Delta f$ measures how much the parton $f$ "remembers" the parent hadron helicity. If the subprocess involves parity violating interactions, one can consider 
another interesting observable which requires only one initial hadron polarized, that is the single helicity asymmetry $A_{L}$, defined as

$$
A_{L}=\frac{d \sigma_{a(-)}-d \sigma_{a(+)}}{d \sigma_{a(-)}+d \sigma_{a(+)}} .
$$

In addition, if both $a$ and $b$ are polarized one can also have two double helicity parity violating asymmetries defined as

$$
A_{L L}^{P V}=\frac{d \sigma_{a(-) b(-)}-d \sigma_{a(+) b(+)}}{d \sigma_{a(-) b(-)}+d \sigma_{a(+) b(+)}} \quad \text { and } \quad \bar{A}_{L L}^{P V}=\frac{d \sigma_{a(-) b(+)}-d \sigma_{a(+) b(-)}}{d \sigma_{a(-) b(+)}+d \sigma_{a(+) b(-)}}
$$

which can be simply related to $A_{L}$ as we will see in section 5 , where we will give their explicit expressions for $W^{ \pm}$production. This completes the presentation of the spin observables and let us now turn to a short discussion on the selected sets of polarized parton distributions.

We first consider the set of polarized parton densities which has been proposed in ref. [9]. The quark and antiquark densities are parametrized in terms of Fermi-Dirac distributions with only nine free parameters. Simple assumptions allow to relate unpolarized and polarized quark distributions and the free parameters are determined from the very accurate data on deep inelastic neutrino scattering at $Q^{2}=3 \mathrm{GeV}^{2}$. For the gluon distributions one uses a Bose-Einstein expression which is given with no additional free parameters. This allows a straightforward DGLAP $Q^{2}$ evolution which leads to excellent predictions for $F_{2}\left(x, Q^{2}\right)$ up to the $x, Q^{2}$ kinematic range accessible at HERA. These quark densities give also a fair description of the low $Q^{2}$ structure functions $g_{1}^{p, n}\left(x, Q^{2}\right)$. However, in this approach $\Delta G\left(x, Q^{2}\right)$ at $Q^{2}=3 \mathrm{GeV}^{2}$ is not known and two possibilities have been proposed, namely the soft and the hard gluon polarization which are shown in Fig.1a.

There is another standard method for obtaining the polarized parton distributions, which is more generally used in the literature. It is based on the direct analysis of $g_{1}^{p, n}\left(x, Q^{2}\right)$, independently of the unpolarized structure functions. A very simple parametrization for the quark densities, of the type $x^{\alpha}(1-x)^{\beta}$, has been proposed in ref. [10]. It was found that for a correct description of the data, in particular the very recent accurate neutron data [11] from SLAC E154 at $Q^{2}=5 \mathrm{GeV}^{2}$, one needs a rather substantial $\Delta G\left(x, Q^{2}\right)$, having also a simple expression as above, which is displayed in Fig.1a.

In ref. 12 the parametrization is based on more complicated expressions of the type $x^{\alpha}(1-x)^{\beta}(1+\gamma \sqrt{x}+\rho x)$ in a similar way as what was used by Martin-Roberts-Stirling to describe the unpolarized quark distributions [13]. Due to our poor knowledge of $\Delta G\left(x, Q^{2}\right)$, they parametrize it in the same way and they explore various possibilities with three different choices of $\gamma_{G}$ and $\rho_{G}$. They conclude that three scenarios A, B and C, shown in Fig.1b are equally acceptable and therefore $\Delta G\left(x, Q^{2}\right)$ is largely undetermined. However their analysis, which is prior to the SLAC E154 neutron data, does not include it. This is also the case for the parametrization proposed in ref.[14], where the polarized 
distributions are constructed from the unpolarized ones, the so called GRV densities [15] by means of a simple multiplicative factor $x^{a}(1-x)^{b}$. Needless to recall that the free parameters are such as to respect the fundamental positivity constraints down to the low resolution scale of this approach namely $Q^{2}=0.23 \mathrm{GeV}^{2}$. In this case, one gets a gluon polarization, shown in Fig.1b, which is half way between the soft and the hard $\Delta G$ of ref.[9].

In addition to these four sets of polarized parton distributions we just briefly discussed, many other different choices have bee presented in the literature [16, 17]. It appears that from the present available data, the valence quark distributions are fairly well determined and rather consistent with each other, unlike for the sea quarks (or antiquarks) and for gluons as we have seen above.

Measurements of polarized deep inelastic lepton nucleon scattering yield direct information on the spin asymmetry

$$
A_{1}^{N} \sim \frac{g_{1}^{N}\left(x, Q^{2}\right)}{F_{1}^{N}\left(x, Q^{2}\right)} \quad(N=p, n, d),
$$

where $F_{1}^{N}\left(x, Q^{2}\right)$ is simply related to the unpolarized structure function $F_{2}^{N}\left(x, Q^{2}\right)$. All data for $Q^{2}>1 \mathrm{GeV}^{2}$ are consistent with a flat $Q^{2}$ behaviour of $A_{1}^{N}\left(x, Q^{2}\right)$ which reflects the fact that the scaling violations of $g_{1}^{N}\left(x, Q^{2}\right)$ and $F_{1}^{N}\left(x, Q^{2}\right)$ are not so different and not distinguishable within experimental errors. The next-to-leading or leading order QCD evolutions leads to $A_{1}^{N}\left(x, Q^{2}\right)$ almost independent of $Q^{2}$ in accordance with experimental observation. To illustrate this fact we show in Fig.2 the results for the proton case $A_{1}^{p}\left(x, Q^{2}\right)$ from ref. [14]. This interesting property will be used in the next section on direct photon production.

\section{$3 \quad$ Direct photon production}

The cross section for direct photon production on $p p$ collisions at high $p_{T}$ is considered as one of the cleanest probe of the unpolarized gluon distribution $G\left(x, Q^{2}\right)$. This is partly due to the fact that the photon originates in the hard scattering subprocess and is detected without undergoing fragmentation. Moreover in $p p$ collisions the quark-gluon Compton subprocess $q G \rightarrow q \gamma$ dominates largely and the quark-antiquark annihilation subprocess $q \bar{q} \rightarrow G \gamma$ can be neglected. Consequently the double helicity asymmetry $A_{L L}^{\gamma}$ (see eq.(4)), which involves in this case only one subprocess, becomes particularly simple to calculate and is expected to be strongly sensitive to the sign and magnitude of $\Delta G\left(x, Q^{2}\right)$. For the Compton subprocess, $\hat{a}_{L L}$ whose expression at the lowest order is given in ref. [8], is always positive and such that $\hat{a}_{L L}\left(\hat{\theta}_{c m}=90^{\circ}\right)=3 / 5$ where $\hat{\theta}_{c m}$ is the center of mass (c.m.) angle in the subprocess.

Before we proceed, we would like to present an approximate expression of $A_{L L}^{\gamma}$ valid at pseudo-rapidity $\eta=0$, where the cross section reaches its largest values. To a good 
approximation we can write

$$
A_{L L}^{\gamma}(\eta=0)=\frac{3}{5} \frac{\Delta G\left(x_{T}, Q^{2}\right)}{G\left(x_{T}, Q^{2}\right)} A_{1}^{p}\left(x_{T}, Q^{2}\right),
$$

where $x_{T}=2 p_{T} / \sqrt{s}, p_{T}$ being the transverse momentum of the outgoing photon and $\sqrt{s}$ the c.m. energy of the reaction $p p \rightarrow \gamma X$. In eq.(9) one should take $Q^{2}=p_{T}^{2}$ and $A_{1}^{p}$ is the deep-inelastic spin asymmetry we discussed above (see eq.(8)). This expression shows clearly the dependence of $A_{L L}^{\gamma}$ on $\Delta G$ and, in order to test its validity, we have compared the exact calculation of $A_{L L}^{\gamma}$, using eqs.(2) and (4) also with $Q^{2}=p_{T}^{2}$, to the above approximation. The results are depicted in Fig.3a for two sets of polarized parton distributions and show the usefulness of this simplified expression (9). $A_{L L}^{\gamma}$ increases with $p_{T}$ due to the rapid growth of $A_{1}^{p}$ with $x$ and follows the sign of $\Delta G$ and its magnitude which is larger in the case of BS-b than for GRSV (see Figs. 1a,b).

In Fig. 3b we show a more complete comparison of the results we obtained for the $p_{T}^{\gamma}$ distribution of $A_{L L}^{\gamma}(\eta=0)$, using the different sets of polarized parton distributions we have discussed in the previous section, with a leading order $Q^{2}$ evolution. Actually we find that the smallest predictions correspond to the sets ref. 112 and ref. 114 which have the smallest $\Delta G(x) / G(x)$. The predictions differ substantially at large $p_{T}$, which corresponds to the region, say around $x=0.4$ or so, where the distributions $\Delta G(x)$ have rather different shapes. We have also indicated the expected statistical errors based on an integrated luminosity $L=800 \mathrm{pb}^{-1}$ at $\sqrt{s}=500 \mathrm{GeV}$, for three months running time. We have evaluated the event rates in the pseudo-rapidity gap $-1.5<\eta<1.5$, assuming a detector efficiency of $100 \%$ and for a $p_{T}^{\gamma}$ acceptance $\Delta p_{T}^{\gamma}=5 \mathrm{GeV} / \mathrm{c}$. We see that up to $p_{T}^{\gamma}=50 \mathrm{GeV} / \mathrm{c}$ or so, $A_{L L}^{\gamma}$ will be determined with an error less than $5 \%$ which therefore will allow to distinguish between these different possible $\Delta G(x)$. For very large $p_{T}^{\gamma}$, the event rate drops too much to provide any sensitivity in the determination of $\Delta G(x)$. In Fig. 4 we display the pseudo-rapidity distribution of $A_{L L}^{\gamma}(\eta)$ at a fixed $p_{T}^{\gamma}$ value. It shows a systematic increase of $A_{L L}^{\gamma}$ for higher $\eta$, already observed in ref. [18], which is partly due to the cross section fall off away from $\eta=0$. This smaller event rate leads also to larger statistical errors, but this measurement is worth performing because it is obviously a non-trivial QCD test.

Let us now give a short discussion of these results and how they can be compared with other recent calculations. First, one may be concerned about higher order QCD corrections, to our dominant subprocess $\vec{q} \vec{g} \rightarrow q \gamma$. The corresponding K-factors have been calculated [19, 20|⿴囗⿰丿㇄口 and they always exceed 1, so they rescale up separately all spin-dependent cross sections. However this effect tends to be marginal on $A_{L L}^{\gamma}$ which is a ratio of cross sections. Next we should consider the effect of a $Q^{2}$ evolution of the polarized parton distributions which were done here at the leading order (LO) while in ref. 21] they took the next-to-leading order (NLO) version. It is known that there is almost no difference for the valence $(u$ and $d$ ) quark distributions, but this is not the case

1 The results obtained in ref.[20], which are slighty different from those of ref. [19], are based on the choice of a consistent scheme. 
for the gluons. Actually at the NLO, the maximum of $\Delta G(x) / G(x)$ is shifted towards smaller $x$ values and therefore the rise of $A_{L L}^{\gamma}$ is sharper. This is precisely what we see by comparing the GS sets in Fig.3b with Fig.4 of ref. [21], which also uses ref. [12]. Finally there is another aspect to be mentioned. At collider energies the outgoing photon is very often produced inside an hadronic jet, whose energy has to be restricted by isolation cuts in order to improve the detection accuracy. This is a standard procedure experimentally but not at all straightforward theoretically. It has been studied at the NLO with a MonteCarlo method [22] and it was found that the effects of isolation cuts are rather important for unpolarized cross sections, but not so much for $A_{L L}^{\gamma}$ and they decrease with increasing energy. Actually they become minute at $\sqrt{s}=500 \mathrm{GeV}$ and the predictions one finds in ref. 22 are almost identical to those of ref. 21] where isolation cuts were ignored.

\section{Single jet production}

Inclusive jet production is also a physics area where one can learn a lot about parton densities and, considering the vast amount of unpolarized existing data, it has been regarded as an important QCD testing ground. Event rates are much larger than for prompt photon production, but there is a drawback because many subprocesses are involved, unlike in the previous case. In principle one should take into account gluon-gluon $(G G)$, gluon-quark $(G q)$ and quark-quark $(q q)$ scatterings. Although these subprocesses cross sections are not so much different, after convolution with the appropriate parton densities (see eq.(2)), they lead to very distinct contributions to the hadronic spin-average cross section.

In the spin dependent case where no data exist so far, the same ingredients can be used to test earlier determinations of the polarized parton densities and to check the consistency of our picture of the nucleon spin. A first attempt in connection with RHIC was proposed in ref. 18, where various aspects of jet physics were discussed. Here we will restrict ourselves to the double helicity asymmetry $A_{L L}^{\text {jet }}$ for single jet production and in order to clarify the interpretation of our results below, let us recall some simple dynamical features. In the very low $p_{T}^{\text {jet }}$ region, say $p_{T}^{\text {jet }} \sim 10 \mathrm{GeV} / \mathrm{c}$ or so, $G G$ scattering

dominates by far, but its contribution drops down very rapidly with increasing $p_{T}^{\text {jet }}$. In the medium $p_{T}^{\text {jet }}$ range, say $20 \mathrm{GeV} / \mathrm{c}<p_{T}^{\text {jet }}<80 \mathrm{GeV} / \mathrm{c}$ or so, $G q$ scattering dominates and then decreases for large $p_{T}^{\text {jet }}$, to be overcome by $q q$ scattering. Of course these are rough qualitative considerations and accurate numerical estimates for the relative fractions of these different contributions depend strongly on the parton densities one uses.

Let us first look at $A_{L L}^{\text {jet }}(\eta=0)$ and from the above discussion we see that one does not expect an approximate expression similar to eq.(9). However in the medium $p_{T}^{\text {jet }}$ range where $G q$ scattering dominates, $A_{L L}^{j e t}(\eta=0)$ should have a trend similar to $A_{L L}^{\gamma}(\eta=0)$, with perhaps a magnitude reduced by a factor two, since about half of the jet cross section is due to $G G$ and $q q$ scatterings. This is what we see approximately in Fig. 5, where we present the numerical results for $A_{L L}^{j e t}(\eta=0)$ at $\sqrt{s}=500 \mathrm{GeV}$, which should be compared to Fig.3b. We have also indicated the statistical errors which are extremely small in this 
case, because of the huge event rates. The $\eta$ distribution of $A_{L L}^{j e t}(\eta)$ for $p_{T}^{\text {jet }}=60 \mathrm{GeV} / \mathrm{c}$ is shown in Fig. 6 and it is either flat or decreasing for increasing $\eta$. This is at variance with the prompt photon case (see Fig.4), because although the unpolarized jet cross section decreases away from $\eta=0$ ? , the numerator of $A_{L L}^{\text {jet }}(\eta)$ is sensitive to the behaviour of the different contributions $G G, G q$ and $q q$. We find that while $G q$ increases with $\eta$, both $G G$ and $q q$ are decreasing and therefore the precise behaviour of $A_{L L}^{j e t}(\eta)$ depends strongly on their relative sizes. The statistical errors, assuming a realistic $\Delta \eta=0.2$ per bin, will be small enough to allow the extraction of the correct trend from future data. Finally we recall that the predictions of ref. 21] are somehow different from ours, which is certainly due to the use of different sets of polarized parton distributions. As already noted in ref. [21], the sets of ref. [9] and ref. [12 have different flavor decomposition and, as we will see in the next section, $W^{ \pm}$production is certainly a very promising way to improve our knowledge on the $u, d$ flavor separation.

\section{$5 \quad W^{ \pm}$production}

Let us first consider, for the reaction $p p \rightarrow W^{ \pm} X$, the parity violating single helicity asymmetry $A_{L}$ defined in eq.(6). In the Standard Model, the $W$ gauge boson is a purely left-handed object and this asymmetry reads simply for $W^{ \pm}$production

$$
A_{L}^{W^{+}}(y)=\frac{\Delta u\left(x_{a}, M_{W}^{2}\right) \bar{d}\left(x_{b}, M_{W}^{2}\right)-(u \leftrightarrow \bar{d})}{u\left(x_{a}, M_{W}^{2}\right) \bar{d}\left(x_{b}, M_{W}^{2}\right)+(u \leftrightarrow \bar{d})},
$$

assuming the proton $a$ is polarized. Here we have $x_{a}=\sqrt{\tau} e^{y}, x_{b}=\sqrt{\tau} e^{-y}$ and $\tau=M_{W}^{2} / s$. For $W^{-}$production the quark flavors are interchanged $(u \leftrightarrow d)$. The calculation of these asymmetries is therefore very simple and the results are presented in Figs.7a,b at $\sqrt{s}=500$ $\mathrm{GeV}$, for different sets of distributions. As first noticed in ref. [23], the general trend of $A_{L}$ can be easily understood as follows : at $y=0$ one has

$$
A_{L}^{W^{+}}=\frac{1}{2}\left(\frac{\Delta u}{u}-\frac{\Delta \bar{d}}{\bar{d}}\right) \quad \text { and } \quad A_{L}^{W^{-}}=\frac{1}{2}\left(\frac{\Delta d}{d}-\frac{\Delta \bar{u}}{\bar{u}}\right),
$$

evaluated at $x=M_{W} / \sqrt{s}=0.164$, for $y=-1$ one has

$$
A_{L}^{W^{+}} \sim-\frac{\Delta \bar{d}}{\bar{d}} \quad \text { and } \quad A_{L}^{W^{-}} \sim-\frac{\Delta \bar{u}}{\bar{u}},
$$

evaluated at $x=0.059$ and for $y=+1$ one has

$$
A_{L}^{W^{+}} \sim \frac{\Delta u}{u} \quad \text { and } \quad A_{L}^{W^{-}} \sim \frac{\Delta d}{d}
$$

evaluated at $x=0.435$. Therefore these measurements will allow a fairly clean flavor separation, both for quarks and antiquarks, for some interesting ranges of $x$-values. We

${ }^{2}$ Actually this theoretical prediction has never been checked experimentally since the single-jet cross sections are always given for $\eta=0$ assuming a given pseudo-rapidity cut, usually $|\eta|<0.7$ or more. 
see in Fig.7a that $A_{L}^{W^{+}}$, which is driven by the $u$ and $\bar{d}$ polarizations, leads to similar predictions for all cases. This is mainly due to our knowledge of $\Delta u / u$, except for $x \geq 0.3$ where it comes out to be larger for BS-h [9]. In Fig.7b, for $A_{L}^{W^{-}}$which is sensitive to the $d$ and $\bar{u}$ polarizations, we see that the various predictions leads to the same general trend, with some difference in magnitude due to a large uncertainty in the determination of $\Delta d / d$. Also BS-h 99 has assumed a larger negative $\Delta \bar{u} / u$ which is reflected in the behaviour near $y=-1$. The statistical errors have been calculated with a rapidity resolution $\Delta y=0.2$ and taking into account only the events from the leptonic decay modes. They are smaller for $W^{+}$production which has larger event rates.

Let us now turn to the parity violating double helicity asymmetries $A_{L L}^{P V}$ and $\bar{A}_{L L}^{P V}$ defined in eq.(7). Their explicit expressions are given in ref. [23] and we recall that in $p p$ collisions they have the following symmetry properties

$$
A_{L L}^{P V}(y)=A_{L L}^{P V}(-y) \quad \text { and } \quad \bar{A}_{L L}^{P V}(y)=-\bar{A}_{L L}^{P V}(-y) .
$$

A priori these asymmetries are independent observables, but if one makes the reasonable assumption $\Delta u \Delta \bar{d} \ll u \bar{d}$ for all $x$, one gets the following relations

$$
A_{L L}^{P V}(y)=A_{L}(y)+A_{L}(-y) \quad, \quad \bar{A}_{L L}^{P V}(y)=A_{L}(y)-A_{L}(-y) .
$$

Therefore these double helicity asymmetries do not contain any additional information, but they help to emphasize the differences among the four predictions for $A_{L}^{W^{+}}$and $A_{L}^{W^{-}}$, as seen in Fig.8a,b for $A_{L L}^{P V}$. From an experimental viewpoint, they might be also more useful, if one does not have such a small rapidity resolutions.

Finally let us consider the realistic possibility of having proton-neutron collisions at RHIC, which can be achieved with either deuteron or helium-3 beams. The parity violating single helicity asymmetry $A_{L}$ for $W^{+}$production in $p n$ collisions reads 24

$$
A_{L}\left(\vec{p} n \rightarrow W^{+} ; y\right)=\frac{\Delta u\left(x_{a}, M_{W}^{2}\right) \bar{u}\left(x_{b}, M_{W}^{2}\right)-\Delta \bar{d}\left(x_{a}, M_{W}^{2}\right) d\left(x_{b}, M_{W}^{2}\right)}{u\left(x_{a}, M_{W}^{2}\right) \bar{u}\left(x_{b}, M_{W}^{2}\right)+\bar{d}\left(x_{a}, M_{W}^{2}\right) d\left(x_{b}, M_{W}^{2}\right)},
$$

assuming the proton is polarized. Of course one has a similar expression for $W^{-}$production. The double helicity asymmetries are also related to the sum or the difference of two $A_{L}$ but this time for $A_{L L}^{P V}$, instead of eq.(15), we have the sum

$$
A_{L L}^{P V}\left(\vec{p} \vec{n} \rightarrow W^{+} ; y\right)=A_{L}\left(\vec{p} n \rightarrow W^{+} ; y\right)+A_{L}\left(\vec{p} n \rightarrow W^{-} ;-y\right)
$$

and therefore the following symmetry property

$$
A_{L L}^{P V}\left(\vec{p} \vec{n} \rightarrow W^{+} ; y\right)=A_{L L}^{P V}\left(\vec{p} \vec{n} \rightarrow W^{-} ;-y\right)
$$

Similarly, we find for $\bar{A}_{L L}^{P V}$ that eq.(17) holds, with the difference instead of the sum, and the symmetry property

$$
\bar{A}_{L L}^{P V}\left(\vec{p} \vec{n} \rightarrow W^{+} ; y\right)=-\bar{A}_{L L}^{P V}\left(\vec{p} \vec{n} \rightarrow W^{-} ;-y\right)
$$

Therefore eq.(17) allows us to calculate $A_{L L}^{P V}$, even if one cannot have polarized neutrons. We show in Figs.9a,b the four predictions as in the previous cases ${ }^{2}$.

\footnotetext{
${ }^{3}$ We realize that since neutron beams are not directly available, but they require deuteron or helium-3 beams, $\sqrt{s}=500 \mathrm{GeV}$ is obviously too high to be accessible at RHIC.
} 


\section{Concluding remarks}

In this paper we have briefly reviewed the specific properties of some sets of polarized parton distributions which have been considered in the recent literature, to describe polarized deep-inelastic scattering data. We have stress the importance of the future polarized $p p$ collider at the BNL-RHIC, for studing these distributions, in a kinematic range so far inexplored and to provide the first serious tests of the QCD spin sector.

We have seen that because of the high luminosity of the machine, we will be able to pin down the magnitude and the sign of $\Delta G\left(x, Q^{2}\right)$ which is, at present, the less wellknown distribution. This will allow to verify directly one possible interpretation of the observed violation of the Ellis-Jaffe sum rules [2] for proton and neutron, in terms of the axial anomaly. For this particular topic, one requires detailed measurements of the double helicity asymmetry $A_{L L}$ for single jet and direct photon production. These will be fairly accurately determined as we have dicussed from the small size of the expected statistical errors.

The copious production of $W^{ \pm}$gauge bosons at $\sqrt{s}=500 \mathrm{GeV}$ will also provide an extremely valuable source of information on these distributions and in particular will help us to perform a clean flavor separation between $u$ and $d$ quarks and similarly for antiquarks $\bar{u}$ and $\bar{d}$. This can be achieved from the measurement of the single helicity asymmetry $A_{L}$, but we have also discussed some interesting features of the double helicity parity violating asymmetries $A_{L L}^{P V}$ and $\bar{A}_{L L}^{P V}$ which are simply related to $A_{L}$. Although $W^{ \pm}$production will be easier in $p p$ collisions, we have also considered some interesting aspects of the parity violating asymmetries in the plausible situation where we might have $p n$ collisions.

\section{Acknowledgments}

We thank C. Bourrely and P. Taxil for very helpfull discussions, G. Bunce and M. J. Tannenbaum for valuable informations, and all the participants of the RSC Annual Meeting held in Marseille. This work has been partially supported by the EC contract CHRXCT94-0579.

\section{References}

[1] J. Ashman et al., Phys. Lett. B206 (1988) 364 ; Nucl. Phys. B328 (1989) 1.

[2] J. Ellis and R.L. Jaffe, Phys. Rev. D9,(1974) 1444 ; Erratum ibid D10,1669.

[3] Proceedings of the 12th International Symposium on High Energy Spin Physics (SPIN 96), Amsterdam, september 1996 and references therein. 
[4] J.D. Bjorken, Phys. Rev. 148 (1966) 1467 ; ibid D1 (1970) 1376.

[5] M. Anselmino, A. Efremov and E. Leader, Phys. Rep. 261 (1995) 1.

[6] H.Y. Cheng, Int. J. Mod. Phys. A11 (1996) 5109.

[7] B. Lampe and E. Reya, preprint MPI-PhT/96-14 (to be published in Rev. Mod. Phys.).

[8] C. Bourrely, J. Soffer, F.M. Renard and P. Taxil, Phys. Reports,177, (1989) 319, and the errata preprint CPT - 87/P.2056 (January 1991); see also P.Taxil, Riv. Nuovo Cimento, Vol. 16 (1993), No. 11, 1.

[9] C. Bourrely, and J. Soffer, Nucl. Phys. B445, (1995) 341.

[10] C. Bourrely, F. Buccella, O. Pisanti, P. Santorelli and J. Soffer, preprint CPT96/PE.3327 (revised version december 1996).

[11] E. Hughes (SLAC E154 Collaboration) talk presented at the ICHEP, Warsaw, July 1996 and K. Abe et al., preprint SLAC hep-ex/9705012 to be published in Phys. Rev. Lett.

[12] T. Gehrmann and W.J. Stirling, Phys. Rev. D53, (1996) 6100.

[13] A.D. Martin, R.G. Roberts and W.J. Stirling, Phys. Lett.B354 (1995) 155.

[14] M. Glück, E. Reya, M. Stratmann and W. Vogelsang, Phys. ReV. D53, (1996) 4775.

[15] M. Glück, E. Reya and W. Vogelsang, Phys. Lett. B359, 201 (1995).

[16] H.Y. Cheng and H.H. Liu, Phys. Rev. D53 (1996) 2380.

[17] G. Altarelli, R.D. Ball, S. Forte and G. Ridolfi, preprint CERN-TH/96-345 hepph/9701289) and references therein.

[18] C. Bourrely, J. Ph. Guillet and J. Soffer, Nucl. Phys. B361, 72 (1991).

[19] A.P. Contogouris, B. Kamal, Z. Merebashvili and F.V. Tchakov, Phys. Lett. B304 (1993) 329 ; Phys. Rev. D48 (1993) 4092 and Erratum Phys. Rev. D54 (1996) 7081.

[20] L.E. Gordon and W. Vogelsang, Phys. Rev. D48 (1993) 3136 and ibid D49 (1994) 170 .

[21] R.L. Jaffe and N. Saito, Phys. Lett. B382, (1996) 165.

[22] L.E. Gordon, preprint ANL-HEP-PR-96-100 (december 1996) hep-ph/9612270.

[23] C. Bourrely and J. Soffer, Phys. Lett. B314, (1993) 132.

[24] C. Bourrely and J. Soffer, Nucl. Phys. B423, (1994) 329. 


\section{Figure captions}

Fig. 1a The gluon polarization versus $x$ at $Q^{2}=4 \mathrm{GeV}^{2}$ from ref. [9] (soft gluon BS-s is dotted curve, hard gluon BS-h is dashed curve) and from ref.[10] (BS-i solid curve).

Fig. 1.b The gluon polarization versus $x$ at $Q^{2}=4 \mathrm{GeV}^{2}$ from ref.[12] (gluon A GSA is solid curve, gluon B GSB is dashed curve, gluon C GSC is dotted curve) and from ref.[14] (standard scenario GRSV, dotted-dashed curve).

Fig. 2 The $Q^{2}$ dependence of $A_{1}^{p}\left(x, Q^{2}\right)$ as predicted from ref. [14] at fixed values of $x$.

Fig. 3.a The double helicity asymmetry $A_{L L}^{\gamma}(\eta=0)$ at $\eta=0$ versus $p_{T}^{\gamma}$ for $\sqrt{s}=500$ $\mathrm{GeV}$. The solid curves, which are the exact calculations using BS-h (upper curves) and GRSV (lower curves), are compared with the dotted curves obtained using eq.(9).

Fig. 3b Same as Fig. 3a using BS-h (solid curve), BS-s (large dashed curve), BS-i (dotted curve), GRSV (dashed-dotted curve) GSA, GSB and GSC (small dashed curves).

Fig. 4 Various predictions for the $\eta$ distribution of $A_{L L}^{\eta}$ at $p_{T}^{\gamma}=40 \mathrm{GeV} / \mathrm{c}$ and for $\sqrt{s}=500 \mathrm{GeV}$ (curve labels as in Fig.3b). The statistical errors are calculated for $\Delta \eta=0.5$ per bin and for a $p_{T}^{\gamma}$ acceptance $\Delta p_{T}^{\gamma}=10 \mathrm{GeV} / \mathrm{c}$.

Fig. 5 The double helicity asymmetry $A_{L L}^{\text {jet }}(\eta=0)$ at $\eta=0$ and $\sqrt{s}=500 \mathrm{GeV}$ versus $p_{T}^{j e t}$, for various parametrizations (curve labels as in Fig.3b). The statistical errors are calculated for $\Delta \eta=1$ per bin and a $p_{T}^{\text {jet }}$ acceptance $\Delta p_{T}^{\text {jet }}=10 \mathrm{GeV} / \mathrm{c}$.

Fig. 6 Various predictions for the $\eta$ distribution of $A_{L L}^{\text {jet }}(\eta)$ at $p_{T}^{\text {jet }}=60 \mathrm{GeV} / \mathrm{c}$ and $\sqrt{s}=500 \mathrm{GeV}$ (curve labels as in Fig.3b). The statistical errors are calculated for $\Delta \eta=0.2$ and a $p_{T}^{\text {jet }}$ acceptance $\Delta p_{T}^{\text {jet }}=10 \mathrm{GeV} / \mathrm{c}$.

Fig. 7a Various predictions for the $y$ distribution of $A_{L}^{W^{+}}(y)$ at $\sqrt{s}=500 \mathrm{GeV}$. BS-h (solid curve), GRSV (dashed-dotted curve), GSA (dashed curve), GSC (dotted curve). The statistical errors are calculated for $\Delta \eta=0.2$.

Fig. 7b Same as Fig.7a for $W^{-}$production

Fig. 8a Various predictions for the $y$ distribution of $A_{L L}^{P V}(y)$ for $W^{+}$production at $\sqrt{s}=500 \mathrm{GeV}$ (curve labels as in Fig.7a).

Fig. 8b Same as Fig.8a for $W^{-}$production.

Fig. 9a Various predictions for the $y$ distribution of $A_{L L}^{P V}(y)$ for $W^{+}$production in $p n$ collisions at $\sqrt{s}=500 \mathrm{GeV}$ (curve labels and statistical errors as in Fig.7a).

Fig. 9b Same as Fig.9a for $\bar{A}_{L L}^{P V}(y)$. 


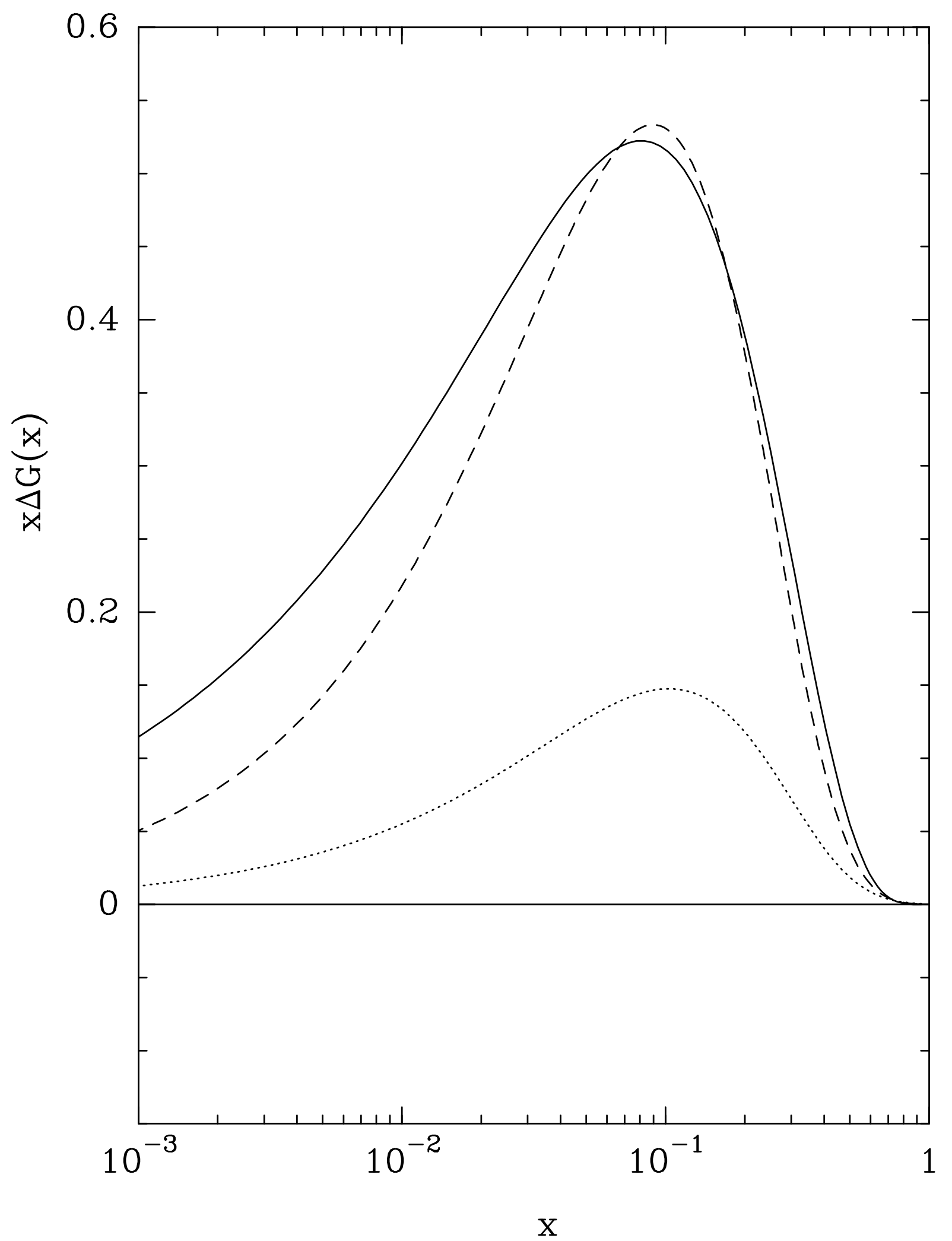

Fig 1a 


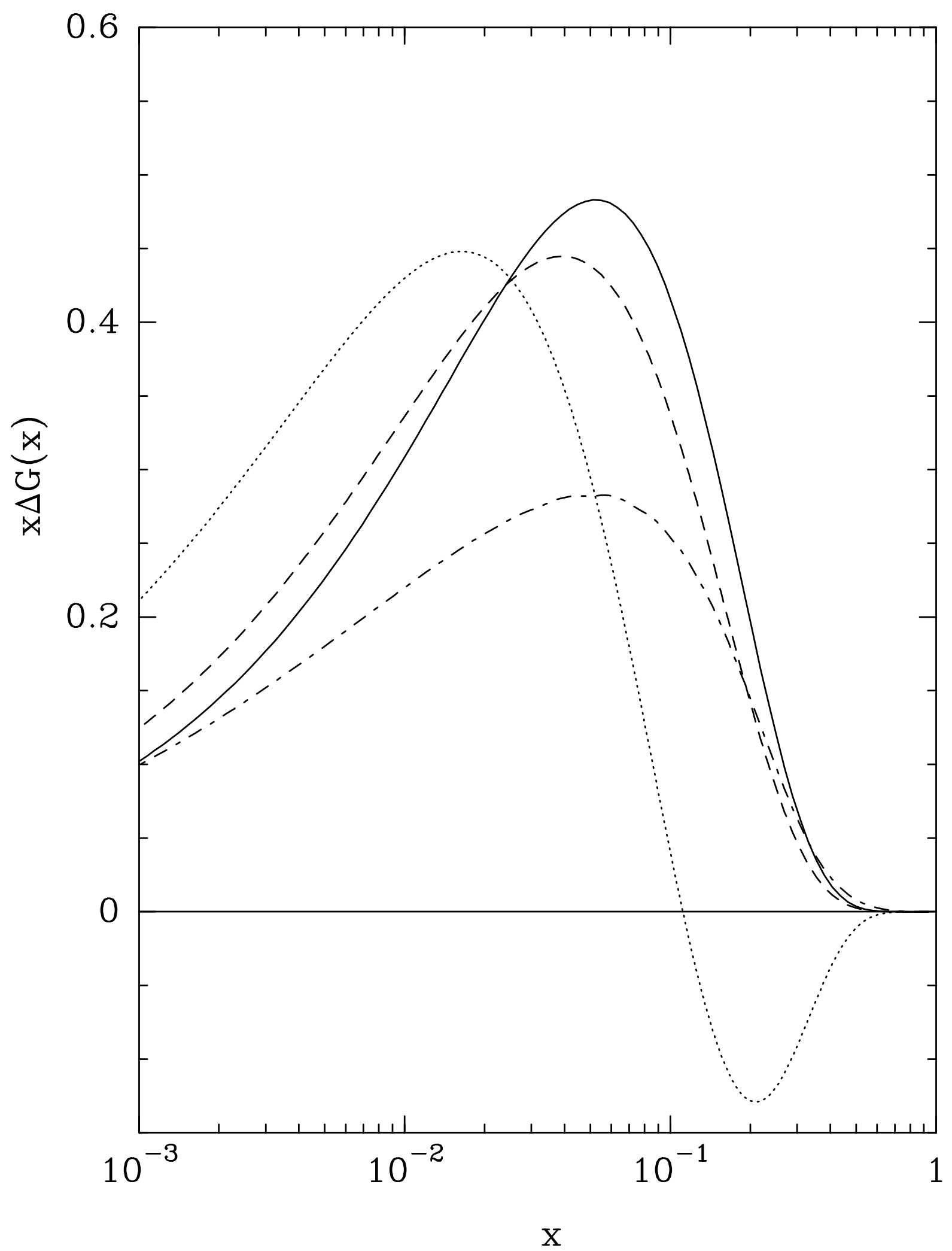

Fig 1b 


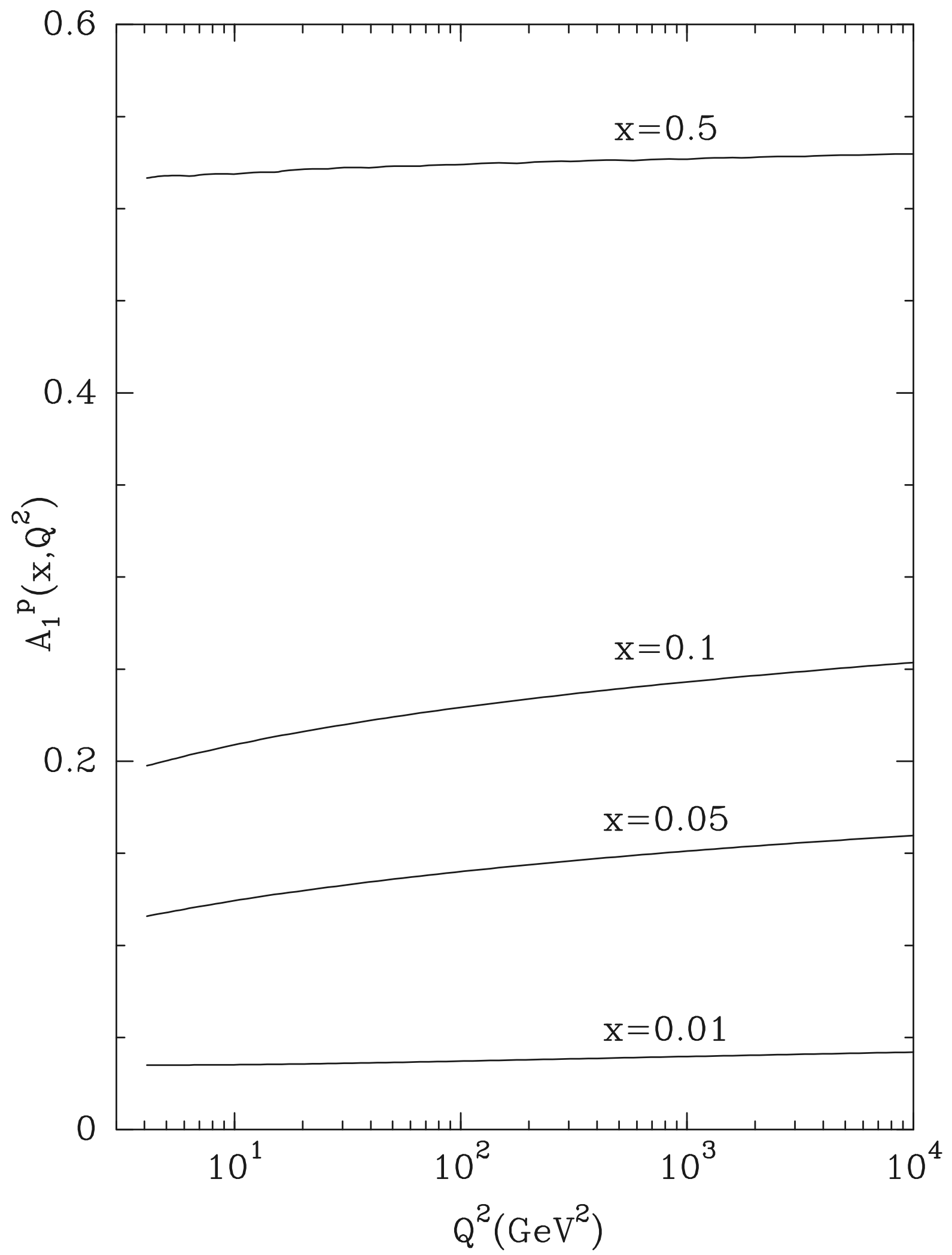

Fig 2 


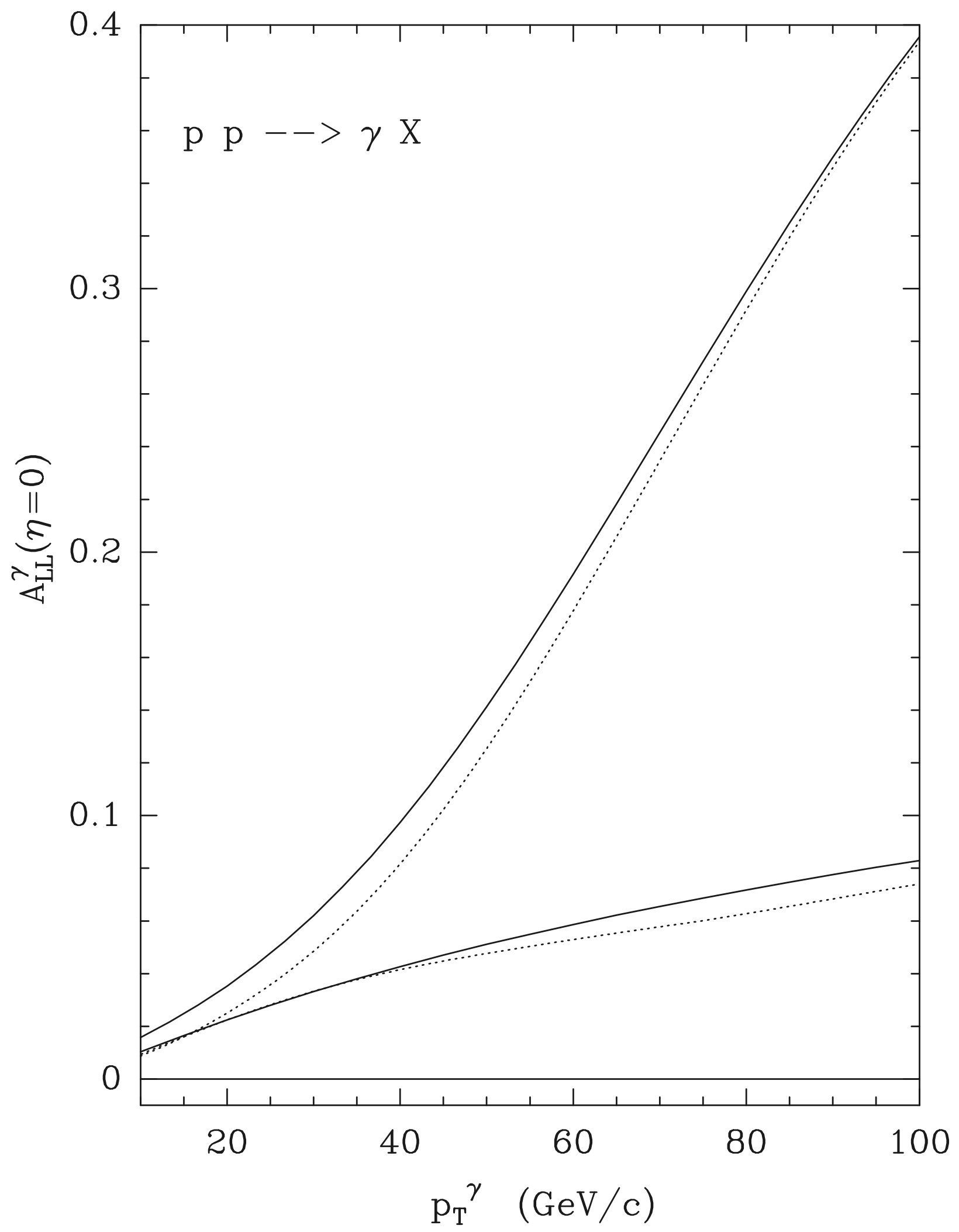

Fig 3a 


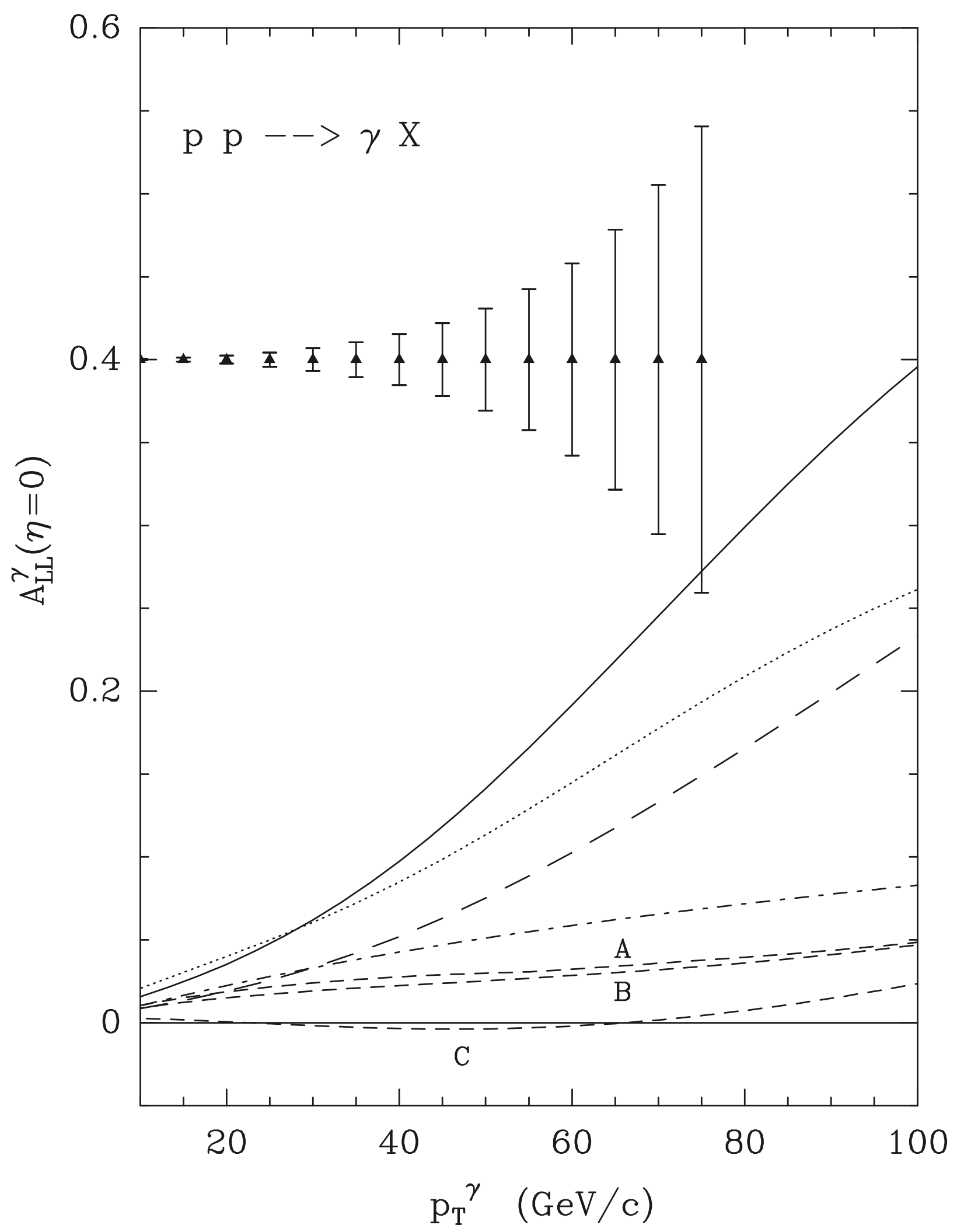

Fig 3b 


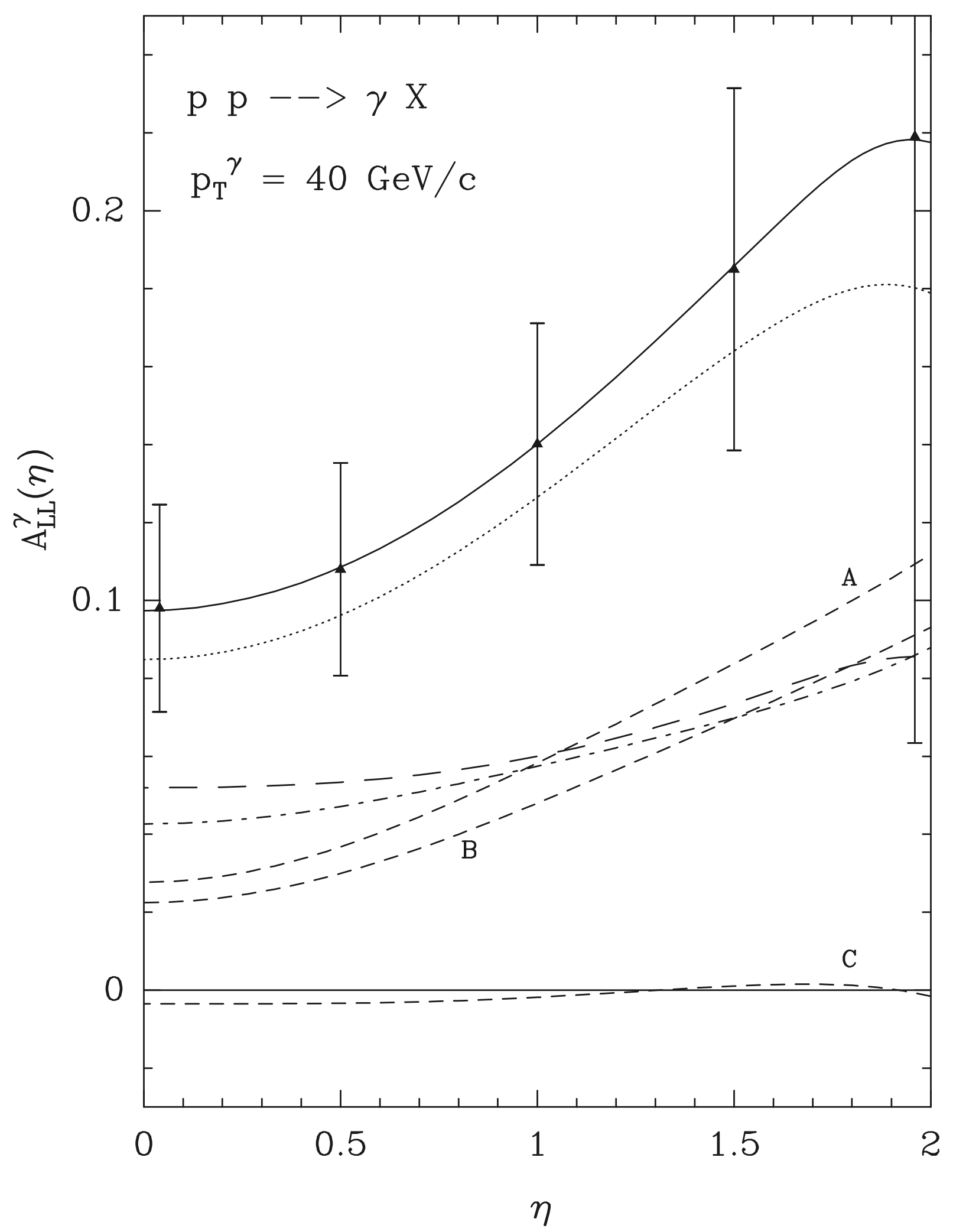

Fig 4 


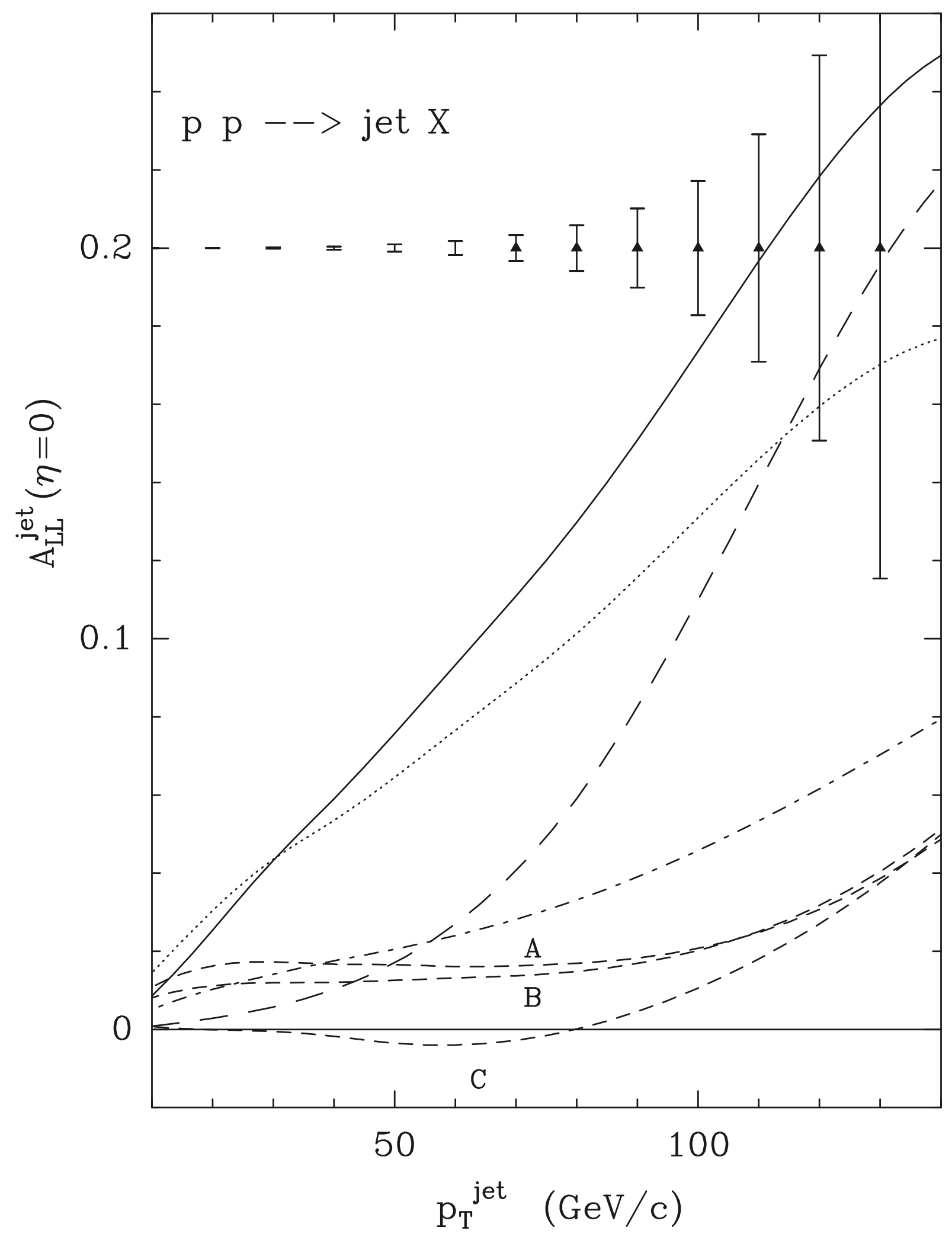

Fig 5 


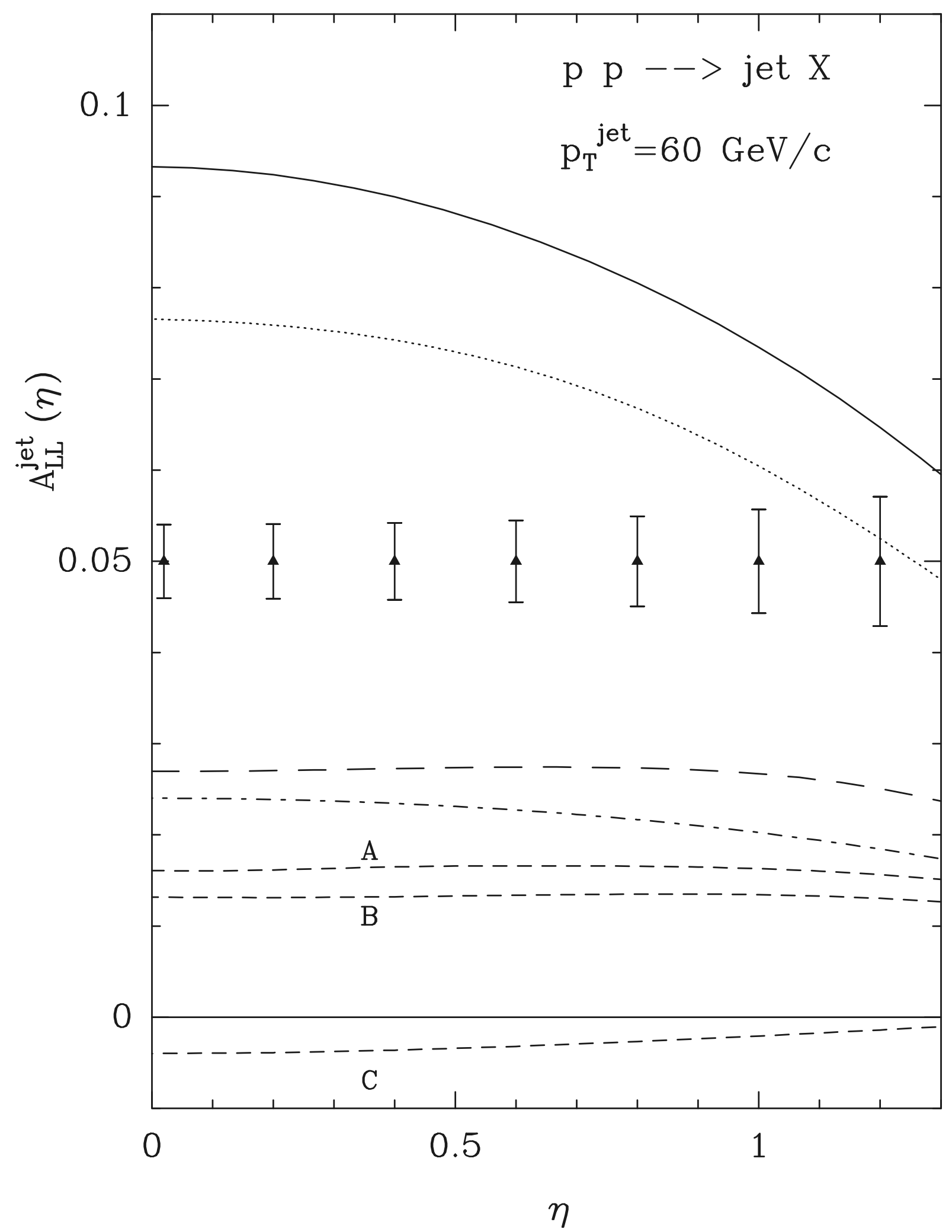

Fig 6 


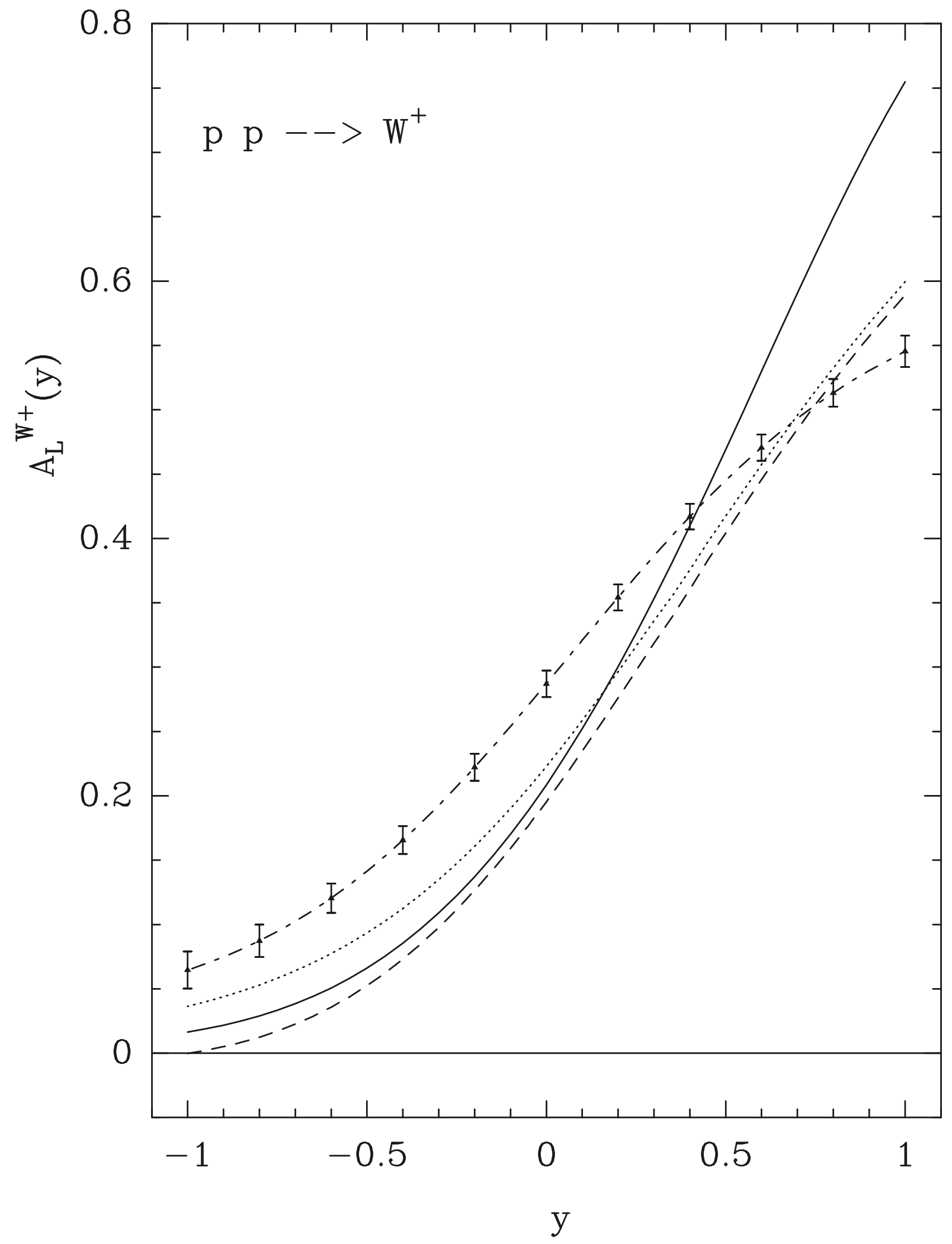

Fig 7a 


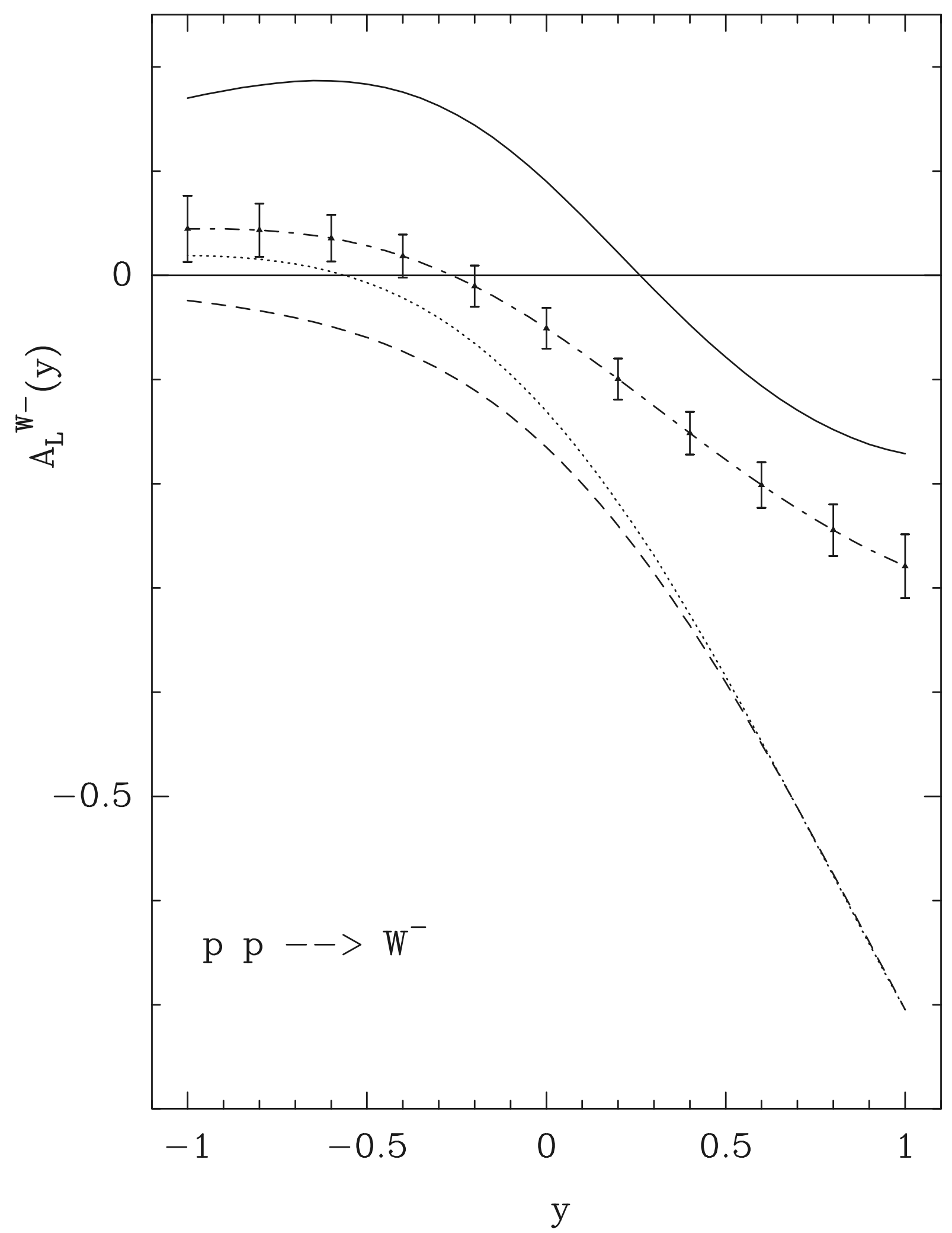

Fig 7b 


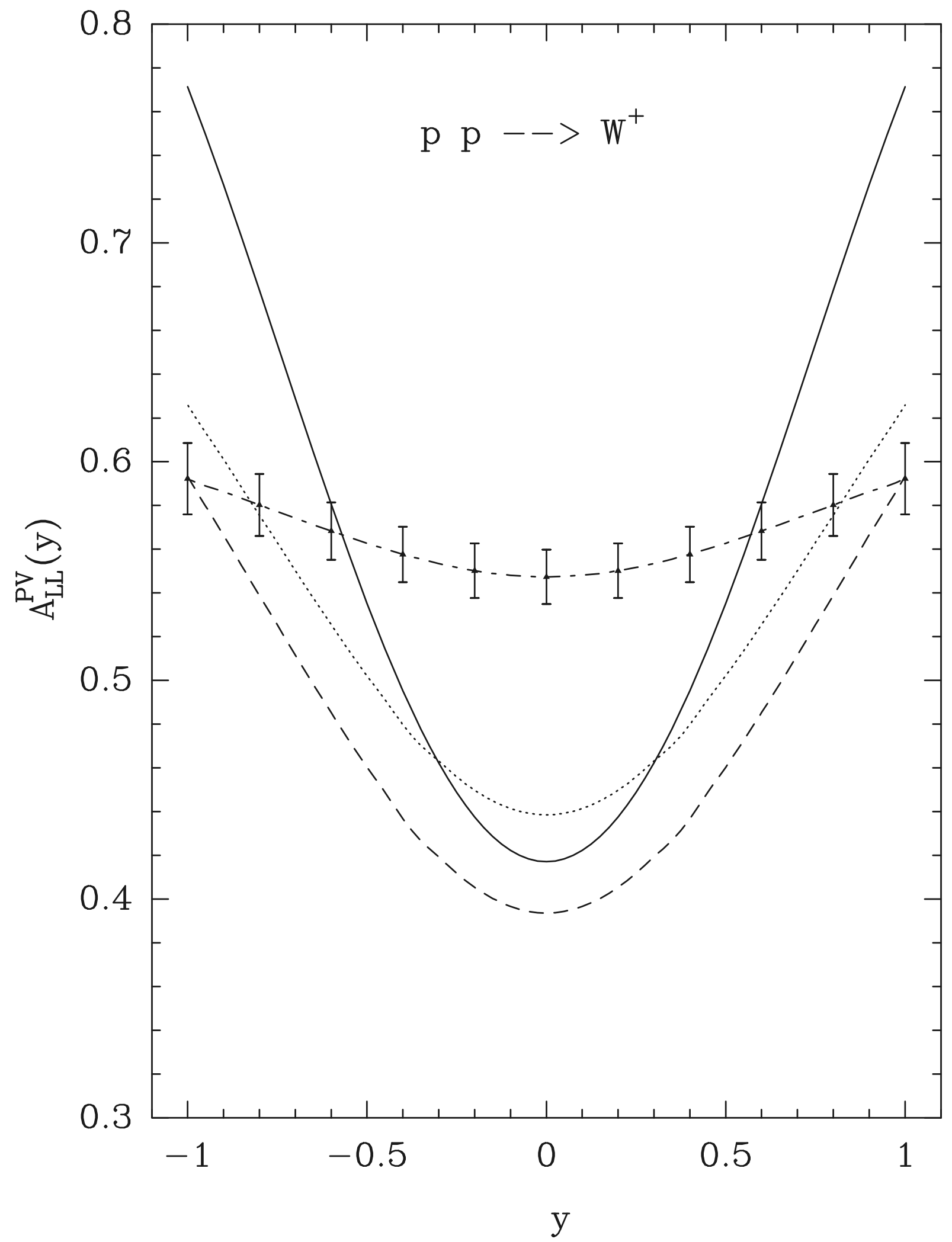

Fig 8a 


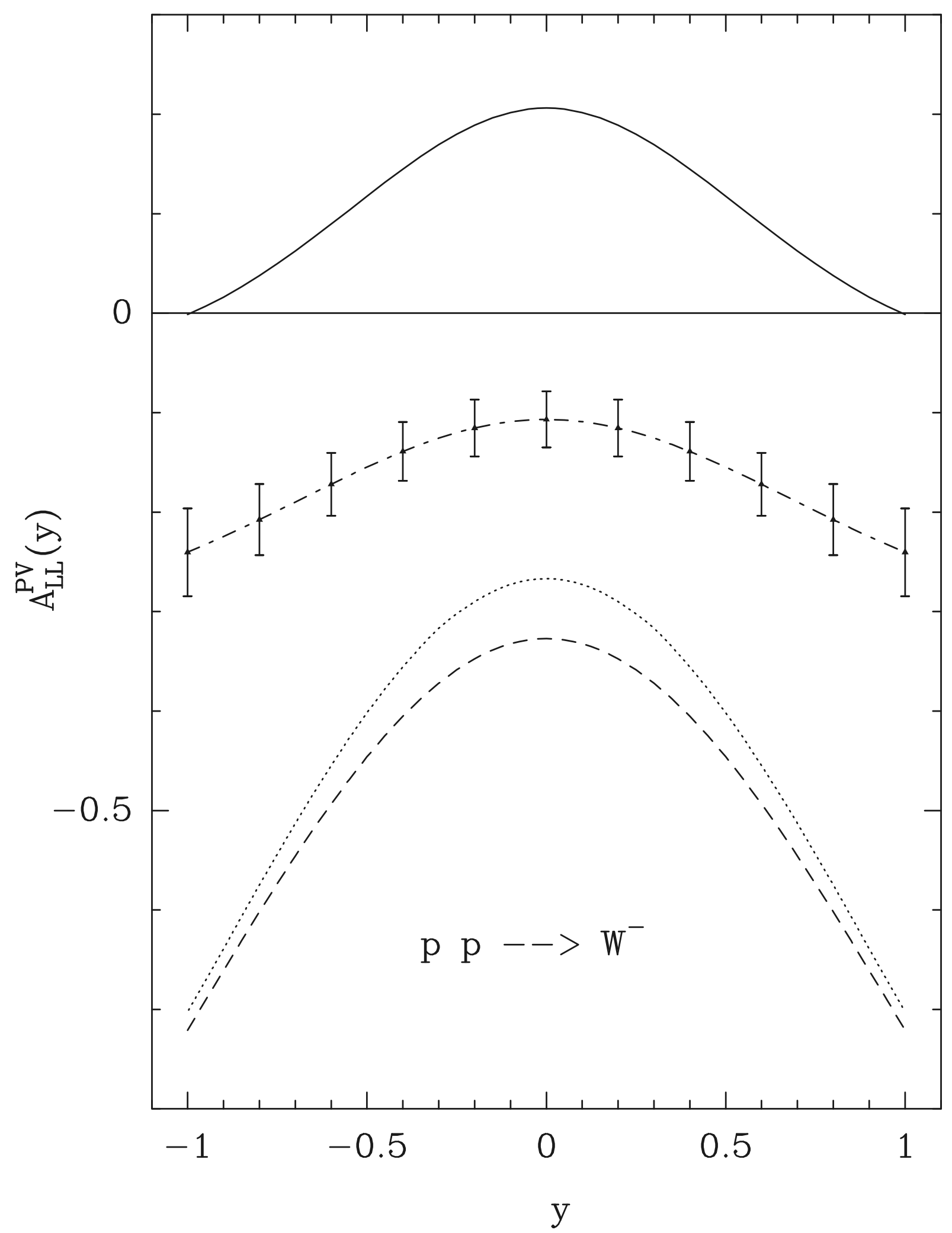

Fig 8b 


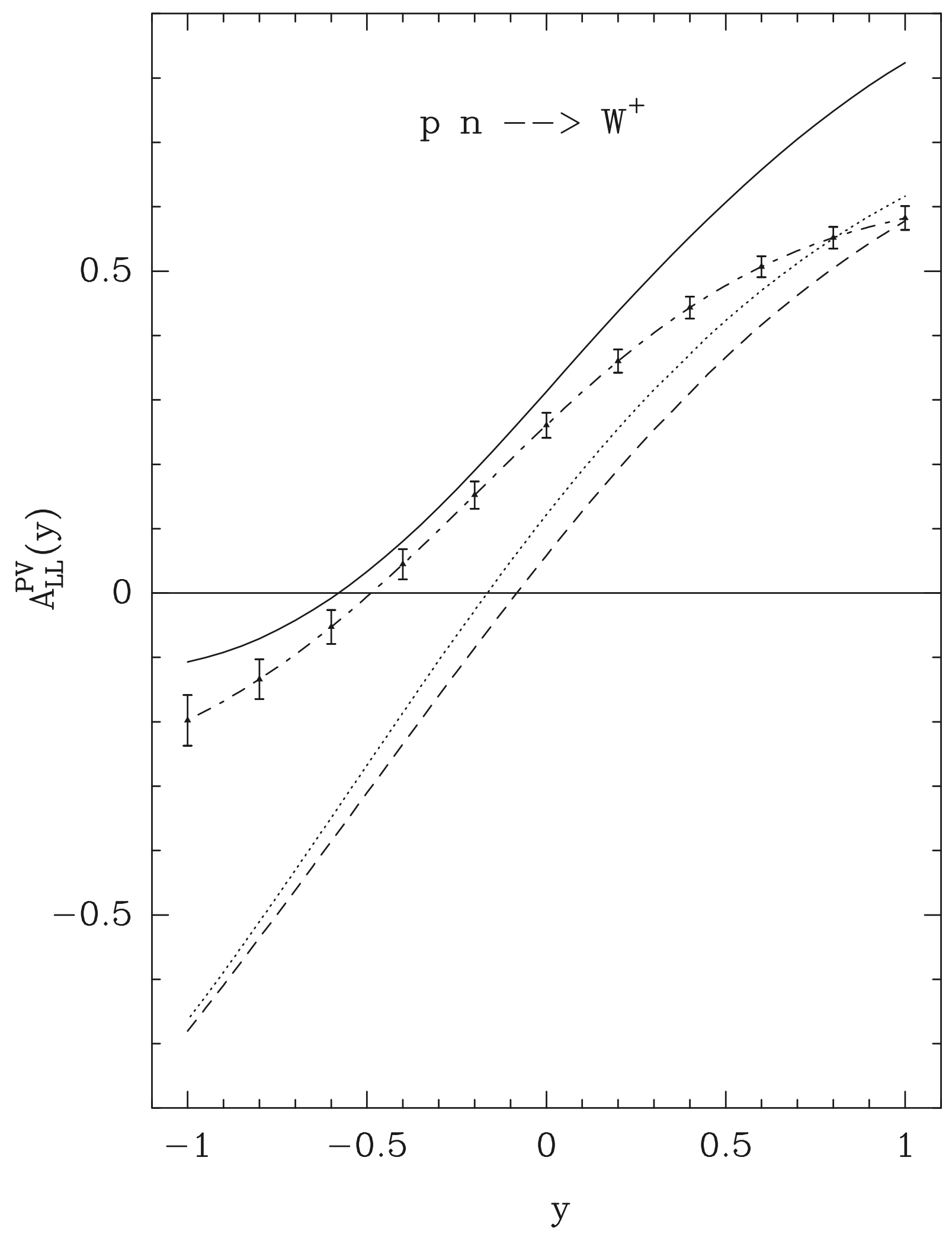

Fig 9a 


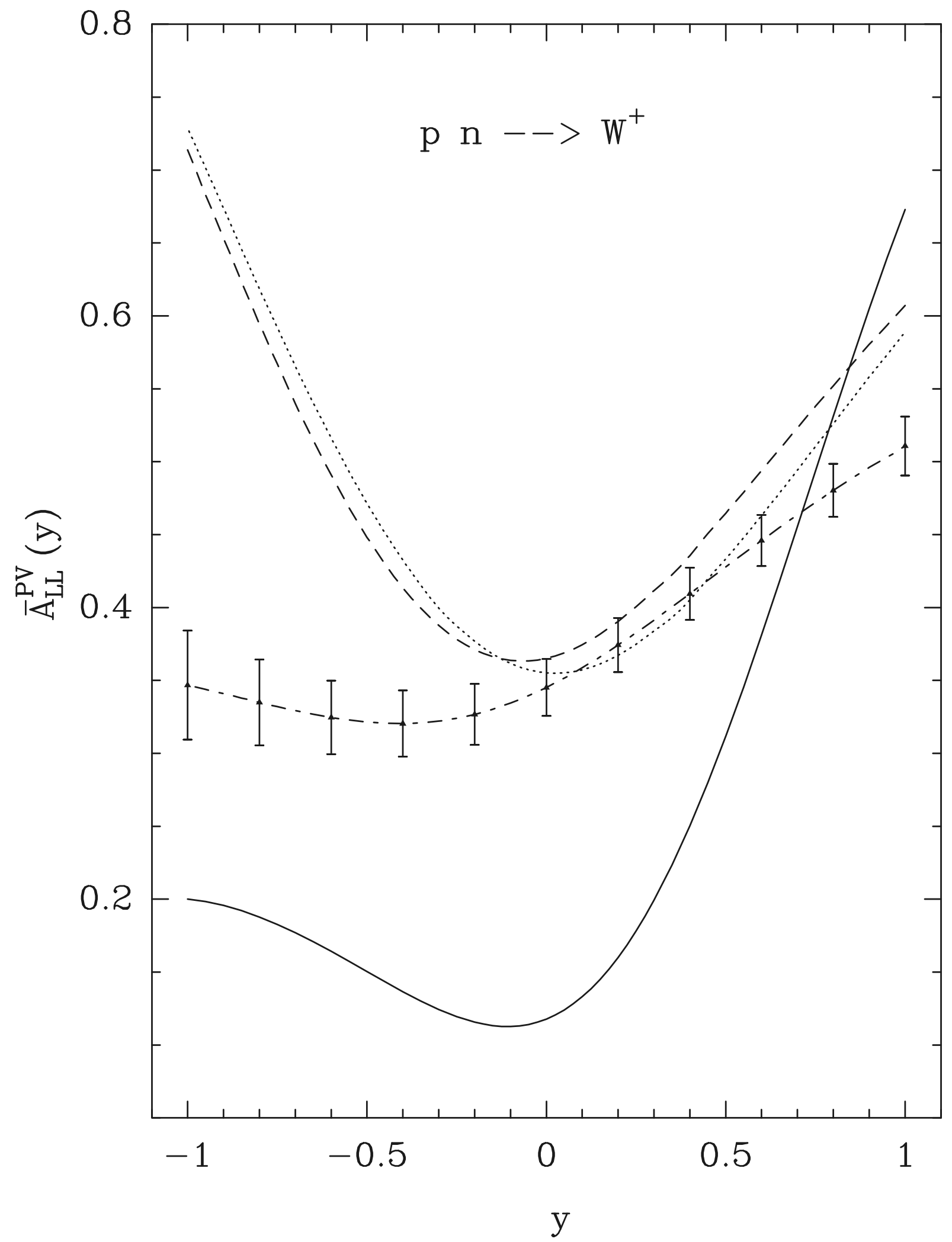

Fig 9b 